\title{
Education packed in technology to promote innovations: Teaching Additive Manufacturing based on a rolling Lab
}

\author{
Laura Katharina Thurn ${ }^{1, *}$, Nicolae Bâlc ${ }^{2}$, Andreas Gebhardt ${ }^{1}$ and Julia Kessler $^{1}$ \\ ${ }^{1}$ Aachen University of Applied Sciences, Faculty of Mechanical Engineering and Mechatronics, \\ Goethestr. 1, 52064 Aachen, Germany \\ ${ }^{2}$ Technical University of Cluj-Napoca, Dept. of Manufacturing Engineering, B-dul Muncii. 103 - \\ 105, 400641 Cluj-Napoca, Romania
}

\begin{abstract}
D Printing is about to revolutionize the way we design and produce future products. This causes not just immense research and development activities worldwide but requires intensive education and training efforts. As 3D Printing is an ideal tool to set up a decentralized production, it is one key to integrate even rural and under-industrialized parts of the country into high tech education and professional training needed. But how to reach the relevant groups - pupils from schools as well as professional staff of preferably companies? Remembered the old proverb: "If the mountain won't come to the prophet, the prophet must go to the mountain" the plan of a mobile 3D printing lab was born. Based on our experiences, we designed the technical infrastructure and the educational approach to adopt Additive Manufacturing courses to a short term comprehensive teaching. To create a fully equipped workplace for every participant, we redesigned a double-decker bus. The paper addresses the strategy behind the bus, the infrastructure in detail, the developed courses taught and the resonance on this project. Looking forward, the paper gives a sneak preview on the further development in terms of specialized courses to be given in the bus as well as in the frame of accompanying activities such as engineering design of parts, application of new materials for Additive Manufacturing such as metals and follow-up production like plastic injection molding.
\end{abstract}

\section{Introduction}

Additive Manufacturing (AM) is the world-wide term for the layer-based technology, which became known as Rapid Prototyping in the late 1980s. Additive Manufacturing, 3D Printing and Desktop Manufacturing are today identical names for those new production technologies that, based on a geometrical representation, creates physical objects by successive addition of material [1] and which are said to be based on a further industrial revolution.

Additive Manufacturing is capable to accelerate the product development by a quickly making complex prototypes with improves quality. AM-processes offer the unique possibility

\footnotetext{
${ }^{*}$ Corresponding author: thurn@,fh-aachen.de
} 
to build complex devices with filigree structures and integrated functionalities and to do this in a resource-efficient way. It is not only an indispensable tool for the direct digital production of models and prototypes, but also a manufacturing process for the production of end products of plastic, ceramics and metal as well as for the production of tools, molds and dies [2].

Everyone involved in design and production, but also in strategic planning, should know about the AM technology to be able to do a qualified assessment and selection.

The application fields of the technology are broad: aerospace, automotive, tool making, medical and electronic engineering, furniture, architecture, design and many more [2].

According to Wohlers Report 2017, the Additive Manufacturing industry, including all AM products and services worldwide, grew $17.4 \%$ (Compound Annual Growth Rate CAGR) to $\$ 6.063$ billion in 2016. This includes revenues from the primary AM market that consists of all products and services directly associated with AM worldwide [3].

\section{Education in the Field of Additive Manufacturing}

Additive Manufacturing, 3D Printing is about to revolutionize the way we think about, design and produce future products. This causes not just immense research and development activities worldwide but requires intensive education and training efforts.

All around the world higher education institutions are developing new curricula and acquiring equipment, software, and materials to support research and instruction. The relatively low prices of desktop 3D printers make them more attractive for training in Today STEM (science, technology, engineering and mathematics) programs at different levels [3].

After initially isolated, informal qualification measures in the enterprises and through trainings from machine manufacturers, today providers of formal advanced training also take up this new technology and offer further training on Additive Manufacturing. The continuing education market as such is made up of offers from the state education system, from institutions close to the economy, such as chambers and associations, from social groups and institutions such as churches, trade unions or foundations, which also provide establishments offering continuing vocational training. In addition, private-sector enterprises with a commercial or non-profit background offer continuing education. Today, In various training programs exist for AM, such as e.g. day seminars, multi-day courses or in-house trainings. In addition, Additive Manufacturing is also integrated into technical advanced training courses, which are not specifically oriented to AM [4].

There is a huge gap between the technical state of the AM technology as a cutting edge technology and present day education that still mostly is done as always. To exploit the whole potential of this new technology that is oriented to practically make things and to remain competitive as an economic (business) location we need to search new ways of teaching.

\section{3 "Upside down" Teaching Approach}

Additive Manufacturing is a typical cross section technology. Education in this field has to include a lot of different disciplines e.g. engineering design, mechanics, electrical engineering, IT, control engineering, programming and materials sciences. In addition, education in the field of AM has to impart a high amount of practical skills.

Teaching new and emerging technologies is not just a multi-disciplinary but also a multi-level task in terms of technical knowledge. The task is not limited to teach how to operate the printer but to teach the necessary computer aided design (CAD) capabilities. Because both design and printing is necessary but time consuming, it is mandatory to keep the target group interested and motivated. For the long-term success, it is inevitable to teach 
not just operating skills, but a deeper involvement in the topic by analysing and feed back to improve the results.

The "GoetheLab for Additive Manufacturing", a research group of the University of Applied Sciences Aachen works in the field of Training on Additive Manufacturing (AM) and has developed, tested and evaluated a wide range of education formats for differentiated target groups.

For the training, we developed an upside down approach, better known as the "v" procedure used for the development of integrated mechanical and electrical systems [5], in our case for both: The CAD, and 3D Printing part. We started with the most complex situation (which mostly is the original), then stripping it down to maximum simplicity and finally working it out to an optimum, compare figure 1.
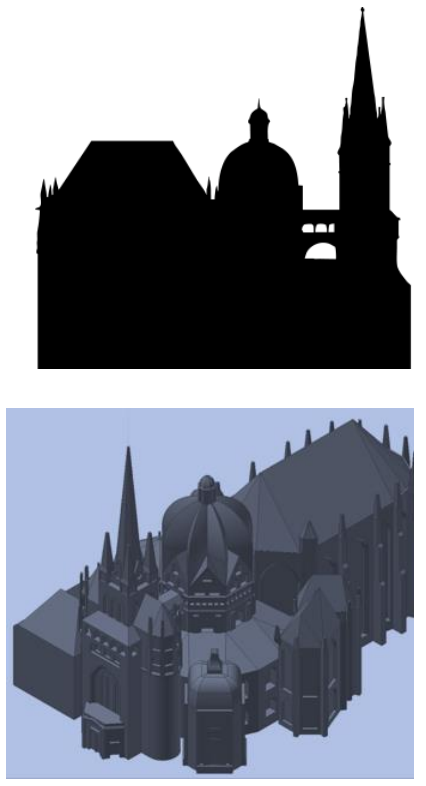
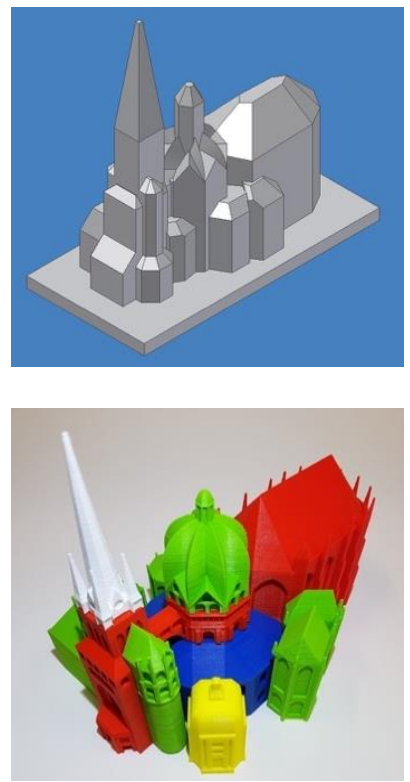
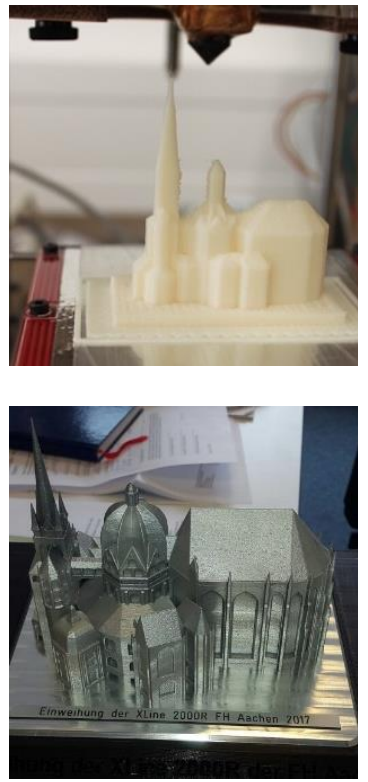

Fig. 1. From 2D drawing to a 3D print; top: left $-2 \mathrm{D}$ silhouette; middle - 3D CAD model; right printed model (personal printer); down: left - complex 3D CAD model; middle - printed model plastic; right - printed model metal.

3D Printing can be taught very effectively if a brought theoretical approach is skipped and the trainees start into the real complex task right from the beginning. This leads to very fast results even accompanied by "brave trail and minor error". As these results are quite rough, the trainees themselves want to improve it and consequently become the driving force.

It turned out that the combination of a direct hands-on course with a comprehensive, theoretical approach to 3D technology meets the demands to teach not just 3D Printing but a wide range of new technologies and is necessary to transfer the knowledge.

This concept can be regarded as an example of how to stimulate social competences by means of technical, interdisciplinary knowledge with Additive Manufacturing: participants gain self-confidence by acting, they experience, give appreciation and learn from/with each other. Simultaneously the enthusiasm for STEM is awakened by an exploratory and playful approach to new technologies [6]. 


\section{Conceptual Design and Development of a mobile Training Lab}

As Additive Manufacturing is an ideal tool to set up a decentralized production, it is one key to integrate even rural and under-industrialized parts of the country into high tech education and professional training needed. But how to reach the relevant groups - pupils from schools as well as professional staff of preferably SME's (Small and medium-sized enterprises)? Extra training, like design and operation of 3D Printers, does not fit in the regular timetable of schools nor can a company accept off-hours of their employees just to reach the training site. Consequently, rural parts of the country are again on the lose end. Small and medium-sized enterprises (SMEs) form the backbone of the economy and especially the middle class outside the metropolitan areas has problems to keep up with the latest findings of science and research. At the same time, SMEs are increasingly lacking technologically advanced specialists to implement new technologies in companies.

Remembered the old proverb: "If the mountain won't come to the prophet, the prophet must go to the mountain" the plan of a mobile 3D printing lab was born. Based on our experiences, we designed the technical infrastructure and the educational approach to adopt Additive Manufacturing courses to a short-term comprehensive Teaching.

The aim is to develop a mobile laboratory facility for flexible use in schools, educational institutions and companies. The lab should be self-sufficient and able to operate a large surface area. To create a fully equipped workplace for every participant, we redesigned a double-decker bus, see figure 2 . The first floor is equipped with a teacher seat and a filament printer on each of the eight pupil seats (see figure 3 - left). The seats consist of eight computer spaces with all required software $(\mathrm{CAD}, \mathrm{AM}$, simulation and printer software). Each floor provides a conference area with enough space to hold meetings or presentations with up to twenty people. The ground floor (figure 3 - right) serves as showroom with industry-level machines and demonstration parts including printed metal parts. The round-up are chill-out-space with literature boards and a coffee bar.
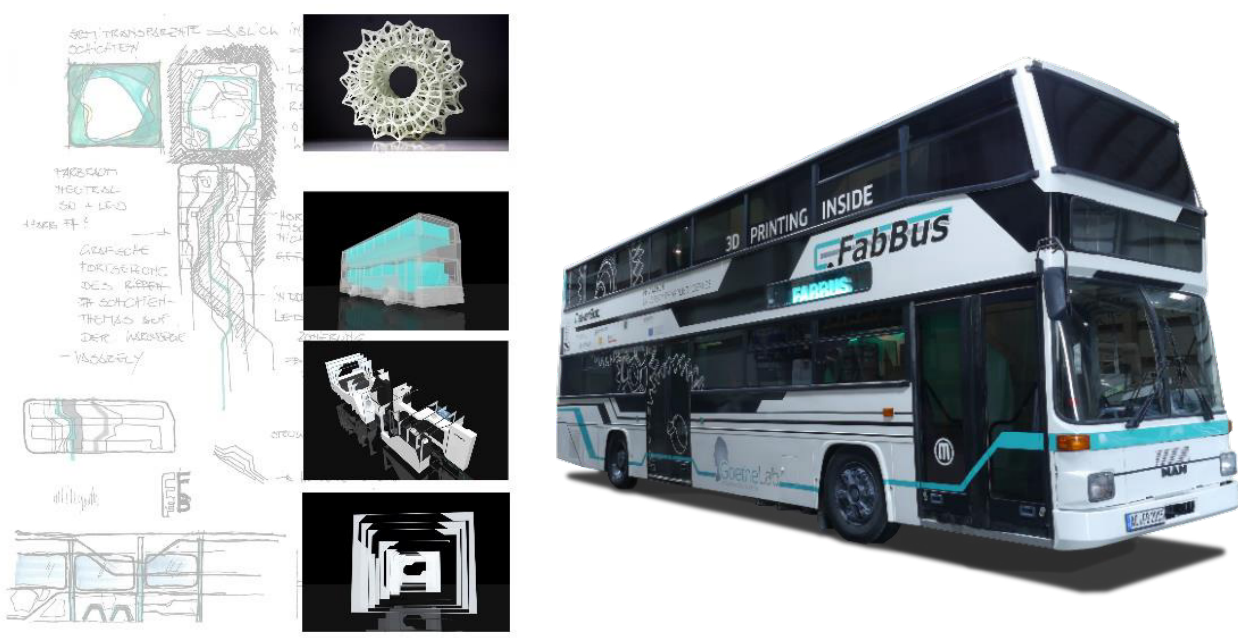

Fig. 2. Left: Design concept (Source: smd partner, bültmann-nießen gbr), right: redesigned double decker bus $-3 \mathrm{D}$ printing inside.

The infrastructure of the bus is designed in such a way that adapted and individualized courses can be offered to address all target groups. The bus bears the name "FabBus - 3D printing inside": the verb "to fab" (from fabricate) has prevailed in the AM scene and stands for printing on small desktop-printers. 

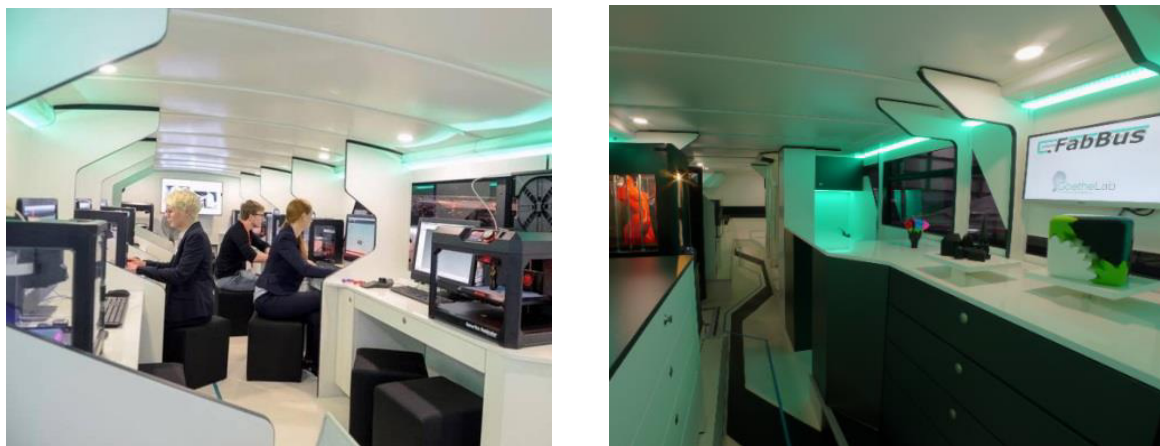

Fig. 3. Left: First floor - Teaching area; right: ground floor - show room and "industry-area".

Especially the local industry will benefit from the mobile lab, in order to present the technology of Additive Manufacturing to their employees and technicians and to enable a "hands-on-training".

\section{Experimental Research on using the FabBus in AM Education}

In May 2015, the bus was inaugurated and has been touring since the borders between Germany and neighbouring countries such as the Netherlands and Belgium. The experience has shown, that completely different target groups express interest and offers, the resonance is huge.

In the period from May 2015 to the end of 2016 more than 50 missions were done using the bus. An internal evaluation showed the following main target groups:

- Schools, universities and other training facilities,

- Small and medium-sized enterprises / corporations / group of companies,

- Trade fairs, congresses and public events.
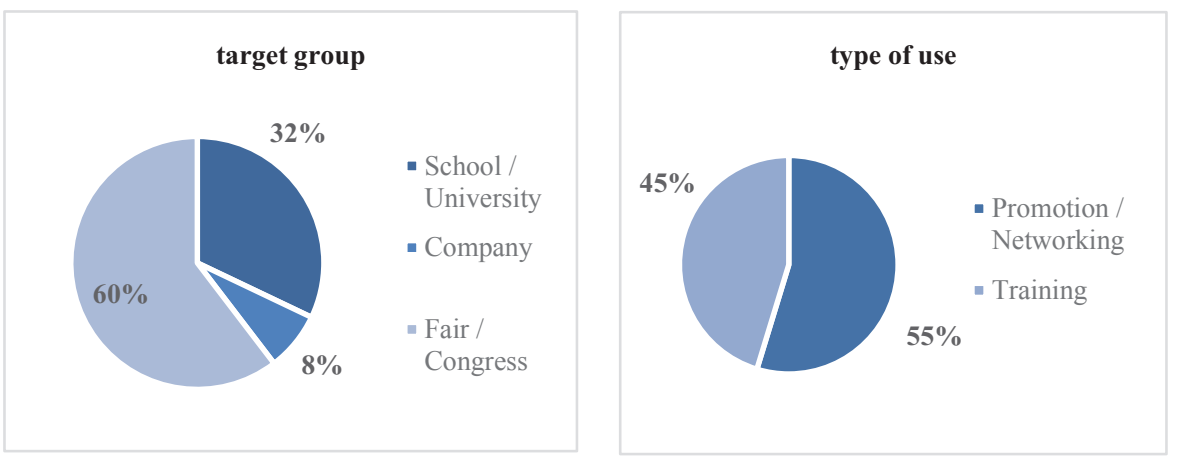

Fig. 4. Percentage distribution of bus operations $(n=53)$ in the period: $05 / 2015$ to $12 / 2016$; Left: Target group distribution, right: Distribution by application type.

There are two main applications for the mobile training lab and a distinction is made between training and promotion/networking. Training includes all events where the bus has been booked to provide training for up to 16 people at a company or at a school / university. Promotion/Networking covers those events where the bus was used as a promoter of innovation, promoter of the AM Technology. Figure 4 gives an overview of the two distributions.

It is striking that $60 \%$ of the events took place at trade fairs and congresses and only $8 \%$ at companies. It should be noted that corporate demand is increasing at the end of the review 
period, which is explained by the growing awareness of AM technology in general, but also by the hypothesis that training in the field of AM is becoming more and more important.

The application areas of AM technology are broad and many different industries are interested in training and further education. Because of this, it is important to offer different formats. To meet the different needs of the various target groups, new (teaching) concepts have been developed. Depending on the experiences of the participants and the desired learning objectives, the following workshops were designed and implemented inter alia with the application of the upside-down approach (compare chapter 3):

- 3D printing and its application $(3 \mathrm{hrs}$.)

- How does a 3D printer work? (3 hrs.)

- From scan to a 3D print (6 hrs.)

- Simple way of 3D construction (6 hrs.)

- Understanding AM in 10 easy lessons (5 days)

- Consulting and Training for the integration of AM in businesses (individual)

- Design for Selective Laser Melting

- Demonstration workshops for beginners and advanced

- Customized Seminars and Workshops

\section{Conclusion}

The rolling laboratory is the core component of a sustainable and future-oriented promotion of young talent and brings Additive Manufacturing (3D Printing) from the laboratories of science to interested people in order to inform them at an early stage and to promote their ideas. With specialist knowledge, the know-how is to be applied to schools and enterprises, so that pupils, employees and technology-oriented people can experience and take advantage of the latest achievements in science and technology. As 3D Printing is an ideal tool to set up a decentralized production, it is one key to integrate even rural and under-industrialized parts of the country into high tech education and professional training needed.

Through a mobile training laboratory, the various needs and the changing (requirements) of training and further education in the field of AM can be addressed. "From lab to fab" is the task of today and from a fixed classroom to a flexible rolling lab is the way how it works.

This research was supported by the OpTi-DeP Project (no. BG101/2016) financed from the UEFISCDI by the Romanian Government and by the HORIZON 2020 AMaTUC project (GA 691787) financed from EU.

\section{References}

1. ISO/ASTM 52900 Standards: Additive manufacturing - General principles Terminology (2015)

2. A. Gebhardt, J. S. Hötter, Additive Manufacturing: $3 D$ printing for prototyping and manufacturing, Carl Hanser Verlag GmbH Co KG, (2016)

3. Wohlers (Wohler's Associates), 3D Printing and additive manufacturing state of the industry: Annual worldwide progress report, (2017)

4. M. Herbert, Personal für die additive Fertigung - Staff for Additive Manufacturing, Springer Verlag, ISBN: 978-3-658-13307-8, (2016)

5. Gerrad Consulting, "The W-Model", https://gerrardconsulting.com/blog/the-w-model/, last visit $5^{\text {th }}$ July (2017)

6. L. Thurn, A. Gebhardt, Arousing Enthusiasm for STEM: Teaching 3D Printing Technology, libreriauniversitaria.it Edizioni (2017) 